\title{
Inverse Methods for Planning Radiation Therapy
}

\author{
A. Brahme \\ Department of Medical Radiation Physics, Karolinska Institute and Stockholm University
}

\begin{abstract}
Modern diagnostic and radiation therapy techniques allowing an entirely three-dimensional approach are leading to the development of new methods for inverse radiation therapy based on calculating the optimum combination of incident beams for a given target volume.
\end{abstract}

A new era in cancer radiation therapy has been gradually emerging during the last decade with the rapid development of many new and powerful methods for planning treatment and for optimizing the delivery of radiation doses. This process parallels in many respects the rapid development in medical imaging technology that took place during the last 20 years, where computed tomography (CT), magnetic resonance imaging (MRI), and single photon and positron emission computed tomography (PET and SPECT) soon matured as universal diagnostic tools for clinical use. However, the parallels between the therapeutic and diagnostic developments are more profound than one might think at first sight.

Both the diagnostic and the therapeutic technologies allow a true three-dimensional approach to be followed along the entire therapeutic chain, from diagnostic imaging to the delivery of the therapeutic effect by accurately shaped radiation beams incident on a tumour. But there are also deeper parallels between the two areas because the mathematical methods used in tomographic image reconstruction are similar to those adopted in some of the new methods for optimizing radiation therapy. This is seen most clearly in the analogy between the non-uniform dose delivery required by most of these methods and the back projection of filtered transmission or emission profiles used in image reconstruction algorithms $[1,2]$.

Owing to the existence of nuclide uptake, a distribution of photon attenuation properties, or a proton density distribution for SPECT, CT or MRI, respectively, these imaging techniques have the advantage that there exists a true solution to the reconstruction problem, at least if all

Anders Brahme, Professor of Radiation Physics at the Karolinska Institute, Box 260, S-171 76 Stockholm, chairs the Department of Hospital Physics, Karolinska Hospital. physical interaction processes such as absorption, scattering and detection noise are taken into account. The problem of radiation therapy optimization is much more difficult because, in general, owing to the laws of Nature most desired dose distributions can never be exactly reproduced by either internal or external radiation therapy sources [2]. It would be, for example, ideal to deliver a high tumour dose and zero dose everywhere else in the body, but this is clearly impossible. From this point of view radiation therapy optimization is thus much more complex than image reconstruction.

\section{Inverse Radiation Therapy Planning}

Mathematically speaking, classical radiation therapy planning is basically a forward process since it tries to answer the question: how will the absorbed dose in the target volume and the surrounding normal tissues be distributed for a given target volume, associated patient geometry and proposed configuration of the inci-

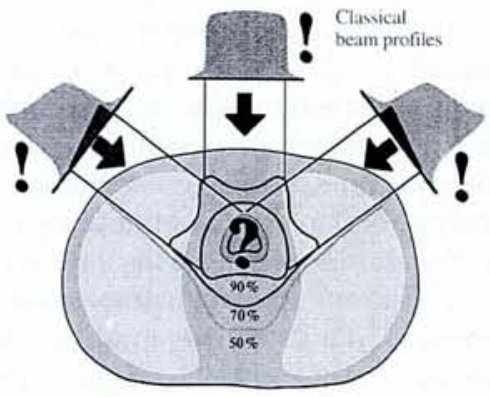

a

Fig. 1. Schematic illustration of the differences between conventional forward radiation therapy planning and inverse planning. Dose optimization using forward calculation based on classical beam profiles (a) is generally a trial-and-error process whereas inverse planning directly results in optimal beam profiles for physically optimal beam profiles (b), and optimal isodose distributions for biological optimal beam profiles (c). The exclamation marks indicate known quantities whereas the question marks indicate the principal unknowns. The contours are for isodoses of $90 \%, 70 \%$ and $50 \%$. dent beams? This is illustrated schematically in Fig. 1a. Optimization of classical radiation therapy is therefore generally a trial-and-error process, where gradually improved dose plans can be found by trying out an increasing number of configurations of the incident beams [3].

By contrast, true radiation therapy planning is fundamentally an inverse problem because what we really want to find out is the optimum combination of incident beams for a given target volume. More exactly, the planning process should answer the question: what configuration and shape of the incident beams are best for controlling growth of a tumour with minimal damage to normal tissue? This question is illustrated in the right-hand panels of Fig. 1. At least for the assumption that either the desired dose to the target volume (Fig. 1b) or the geometrical and radiobiological properties of the patient's tumour and normal tissues are known (Fig. 1c), it should be possible to establish the optimum irradiation method [4].

The conceptual difference between the classical forward calculation and the inverse approach is further clarified by comparing the three panels in Fig. 1. The exclamation marks indicate in each case the known quantities whereas the question marks indicate the principal unknown quantities which need to be calculated (e.g., the optimal isodose distribution in the patient or the optimal incident beam profile). For the inverse calculation, the resulting absorbed dose distribution in the patient is obviously also obtained either by an ordinary forward calculation or by the inversion method itself (Fig. 1c) [5]. From
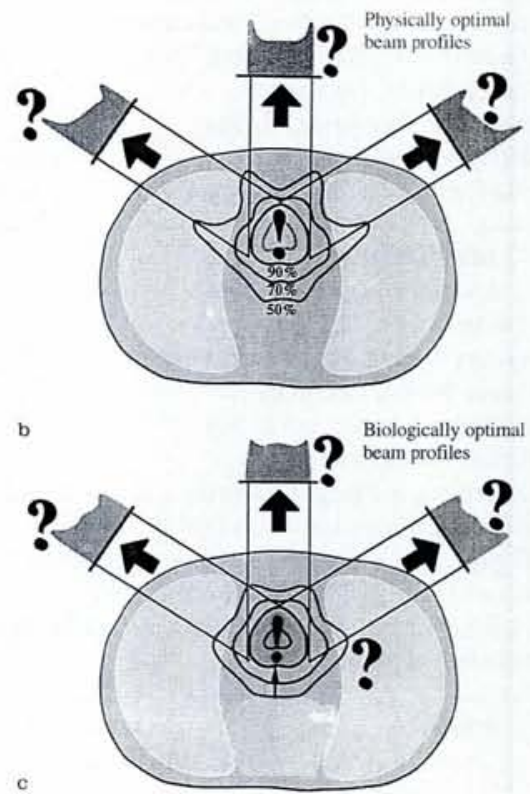
Fig. 1 it is clear that there is some similarity between the inverse problem of radiation therapy and image reconstruction using data taken from a limited number of directions in diagnostic radiology.

\section{The Integral Equation}

The principal problem of radiation therapy planning can be expressed mathematically in the form of an integral equation for the resulting dose distribution in the patient expressed in terms of the incoming radiation field. The most elementary incident radiation beam is a monodirectional beam $p^{m}(E, \Omega, r, Q)$ from a point source, where $p^{m}$ describes the energy deposition at a distance $r$ for a given energy $E$, point $\varrho$, and direction of incidence $\Omega$ for a beam of modality (particle type) $m$ which includes photons (external beams or internal sources), electrons e, protons $\mathrm{p}$, photons $\mathrm{v}$, or neutrons $\mathrm{n}$, as illustrated in Fig. 2. The absorbed dose at the point $r$ in the patient is then given by the integral of the incident particle fluence $\Phi_{E, \Omega}^{m}(\varrho)$ for all particle modalities, energies and angles of the incident beams on the points $\varrho$ on the patient's surface:

$D(r)=$

$\oiint \iiint_{S} \sum_{\Omega m} p^{m}(E, \Omega, r, \varrho) \Phi_{\mathrm{E}, \Omega}^{m}(\varrho) \varrho \mathrm{d} E \mathrm{~d} \Omega d^{2} \varrho$

Here the spatial integrals have to be performed over the relevant entrance surface $S$ of the patient (see Fig. 2 and [6]). From Eq. (1) it is clear that the required energy fluence is implicitly defined by the integral equation when we want to deliver a known dose distribution $D(r)$ using a certain type of radiation with a pencil beam having a known energy deposition dose distribution (kernel) $p^{m}$.

\section{Cylindrical Target Volumes}

Nonlinear wedge techniques for Iymph node irradiation in the head and neck region represented one of the earliest and simplest applications of inversion methods in external beam radiation therapy planning (a related inverse problem for internal sources had been discussed previously by Mayneord and Davison [7]). Using a double-arc method, Lax and Brahme [8] developed a strongly nonlinear wedge filter to give a highly uniform dose to the nodes and low dose to the spinal cord. A numerical solution for the wedge profile was first established using an iterative procedure. An integral equation for rotationally symmetric therapy where the beam moved across an arc was then derived. The desired radial dose profile in the patient $D(r)$ was given by:

$$
D(r)=\oint d(x) \mathrm{e}^{-\mu_{p}} \mathrm{~d} \varphi / \pi
$$

for an effective attenuation coefficient $\mu_{p}$ of the beam and an angle $\varphi$. This equation was based on the simple fact that the dose at a given radius after rotation is equal to the line integral around the same radius in the stationary beam. A Laplace transformation on this integral resulted in a general inversion formula which allowed the calculation of the required beam profile for a given desired dose distribution in the patient assuming cylindrical symmetry and $360^{\circ}$ rotation:

$$
d(x)=\frac{\mathrm{d}}{\mathrm{d} x} \int_{r_{0}}^{x} \frac{\cos \left[\mu_{p}\left(x^{2}-r^{2}\right)^{1 / 2}\right]}{\left(x^{2}-r^{2}\right)^{1 / 2}} D(r) \mathrm{d} r
$$

Here $d(x)$ is again the required dose profile of the incident beam [8]. Unfortunately, most patients are not rotationally symmetric so this general inversion equation is not generally applicable. However, a straightforward generalization of the theory for an arbitrary shape is possible provided the target volume remains cylindrically symmetric. Since the derived dose profiles of the beam given by Eq. (3) pertain to a plane through the centre of the target volume, obtaining the optimum shape of the incident beam at each angle is simply a matter of an attenuation correction for the non-uniform beam absorption in each computer tomographic slice of the body. This obviously results in angularly varying incident-beam profiles for the

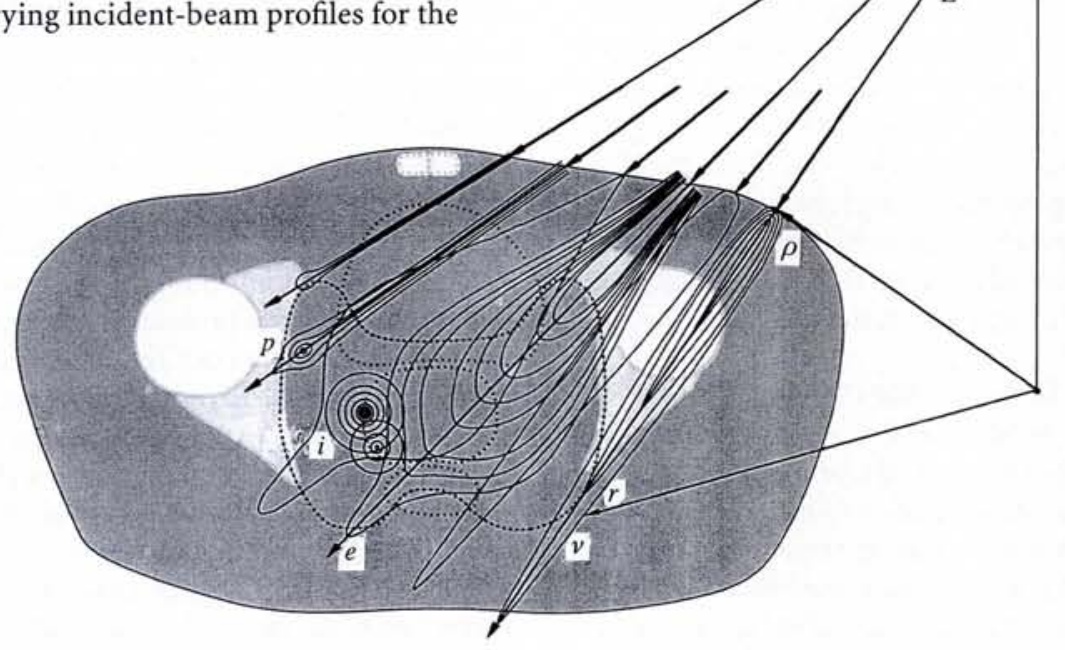

Fig. 2. A schematic illustration of the irradiation geometry used in optimizing the overall dose distribution in the patient following irradiation by a fluence $\Phi_{E, \Omega}^{m}$ of pencil beams sweeping the patient. By using accurately calculated pencil beams an exact optimization is possible on considering all the major constraints to the dose delivery, even after taking inhomogeneities in the patient into account. The point source of the monodirectional beam is at s and $\mathrm{p}^{m}(E, \Omega, r, \varrho)$ describes the energy deposition at a point $r$ for a given beam energy $E$, location $\varrho$ and direction $\Omega$ and type of particle $m$ (electrons e, protons $p$, photons $v$, and internal sources $i$ ). The absorbed dose at $r$ is given by the integral of the fluence over the energy and incident angle, integrated with respect to the entrance surface. general case, even though the target vol-

Unfortunately, Eq. (3) does not result in physically possible incident dose distributions for arbitrary desired dose profiles, $D(r)$, in the patient since in general $d(x)$ will take both negative and infinite values, a problem that has been discussed recently $2,8,9]$. A closely related inversion problines has been solved by Birkhoff in 1940. The striking similarity between both the slice reconstruction problem and Birkhoff's problem and the therapy optimization problem [10] is just now becoming particularly clear. If the uniform lines are and the inverse problem of radiation therapy would be solved. However, even if a solution similar to Eq. (3) can be found it would in general be difficult to use because a non-negative solution does not exist for most desired dose distributions. Furthermore, the requirements given by Birkhoff for the existence of a solution in the drawing case, namely that the coefficients of the resulting Fourier series have to be continuous and form a non-negative continuous function, are already difficult ume is rotationally symmetric. replaced by "pencil" beams then the solution can be generalized for these "lines" 
to apply. Such requirements help little when trying to judge if a desired dose distribution can be produced or not. They are, moreover, useless for finding the best possible solution when an exact solution does not exist. The fact that the requirement of non-negativity has no straightforward useful counterpart in Fourier space was demonstrated in 1948 by Bochner.

\section{Biologically Optimized Treatment}

Fortunately, many different methods for obtaining a fairly good optimization of the dose delivery can be obtained by treating the negativity problem in an approximate way [11]. Approximations can be made by adding a uniform bias or by using iterative methods where the negative portions in the beam profiles are systematically eliminated $[10,12]$. The composite (multi-beam) energy deposition kernel also represents a powerful technique for overcoming the negativity problem while producing physically realizable solutions [11]. These approximate methods generally result in good dose plans, basically because there are many degrees of freedom available for non-uniform dose delivery.

However, as was shown recently [10, 13], for the extremely difficult optimization problems associated with organs at risk close to a tumour, it is essential to use a method which describes the real world as accurately as possible. The pencil-beam method (Fig. 2) with realistic radiobiological dose response data for the tumour and surrounding normal tissue (see Fig. 1) provides the highest accuracy since it allows a direct optimization of the probability of curing the patient without inducing severe damage to normal tissue. Optimization algorithms for pencil beams are today undergoing rapid development; they have the potential to improve the outcome of treatment by $10-20 \%$, especially for advanced tumours that have spread locally in a complex pattern $[2,13,14]$

\section{Modern Dose Delivery Methods}

As illustrated in Fig. 3, there are essentially four methods for increasing the flexibility of dose delivery in external beam radiation therapy (narrow pencil beams; elongated, intensity-modulated fan beams; classical block-collimated beams with a wedge filter; generalised non-uniform beams of irregular cross-section generated, for example, by dynamic multileaf collimation). One can begin by presenting the methods that are available today for non-uniform dose delivery before describing the more "differential" approaches.
Fig. 4. summarizes the principle methods for non-uniform dose delivery. It can be seen that if full dynamic flexibility and reasonable treatment times are required in applying, in the clinic, multidimensional optimization techniques, the best methods for non-uniform dose delivery are dynamic multileaf collimation [15] and scanned elementary beams [5].

Dual dynamic jaw collimation (Fig. 4) also allows, in principle, full modulation of the incident beam, but at the cost of extended treatment times. Furthermore, it requires completely asymmetric upper and lower jaws so that a narrow, rectangular beam spot can be scanned arbitrarily across the entire target volume. If very high dose rates are available and the speed of motion of the collimator jaws is rapid, the time required can be reduced, but this may not be realistic with presently available accelerator systems.

The classical filter and transmission block techniques have sufficient flexibility. But they are fairly time consuming so they are probably impractical for more than three treatment beams per patient. They could, for example, work with the fewbeam techniques indicated in the lower panel of Fig. 1, either by manual manipulation or using a filter revolver at the front end of the treatment head carrying $3-5$ filters. Recent years have seen the development of several techniques for optimizing compensators. They are quite useful for handling few-beam techniques provided suitable beam directions can be identified [16]. In practice, choosing the best beam direction is one of the most difficult problems of treatment optimization since it involves a restriction on the phase space of feasible beam combinations. This selection cannot be achieved without first locating all beam combinations corresponding to local optima, which in practice is equivalent to a global optimization [14]. It also accentuates a serious problem in radiobiology which is in a certain sense the Scylla and Charybdis of radiation therapy: using a single beam, small volumes of normal tissue in the entrance region receive a relatively high local dose, whereas at the other extreme, with a continuum of beams moving over an arc, large volumes receive relatively low doses [17]. Realistic radiobiological objective functions capable of distinguishing between these extremes are needed for a strict optimization [18].

\section{Fan-and pencil-beam therapies}

There are a large number of projects centred around the use of uniform or non-

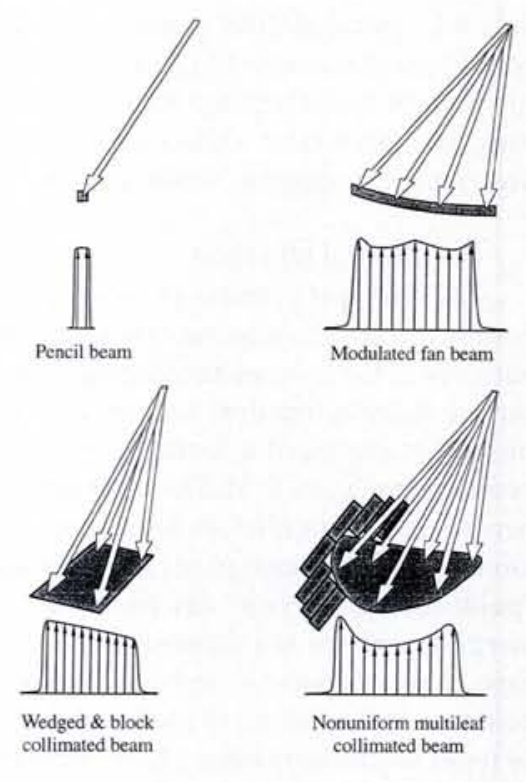

Fig. 3. The four major techniques for increasing the flexibility of dose-delivery in external photon beam radiation therapy. The most precise are pencil-beam methods, either using electromagnetically or mechanically scanned beams, and techniques based on fan beams such as the Peacock device and the tomotherapy method. Classical external beam therapy mainly employs uniform, rectangular block-collimated beams, sometimes with a wedge filter. Fully non-uniform beams can be delivered with any of the methods, and the non-uniform treatment technique is generally the most rapid and simplest to verify.

uniform fan beams (see Fig. 3). The earliest application was probably for computer controlled therapy, where the width of a narrow, elongated slit beam through the rotational axis (the fan beam) was varied as the gantry rotated and the patient was moved slowly through the beam. The treatment time was usually long, of the order of 20 minutes, and the set-up time was also considerable. These problems are common to all small-volume irradiation techniques unless the dose rate and speed of rotation are increased by about one order of magnitude.

A special modulated fan-beam collimator has been developed recently [19]. It allows temporal modulation of the treatment time along the fan beam to provide non-uniform dose delivery. The most recent development, proposed by a group in Madison, USA [20], uses a fan-beam modulating collimator for "spiral irradiation" together with a longitudinally moving patient, in much the same way as is used for spiral computed tomography. However, like all the fan-beam approaches described here, unless the accelerator output is very high the treatment times are unfortunately fairly long 
At the cost of a further increase in treatment time it is possible to use a moving narrowly collimated beam (pencil beam) to deliver non-uniform dose distributions. The pioneering work, reported in 1963 , for uniform beam delivery was carried out in Chicago using a mechanically moving bending magnet in a rotary gantry. Since the dose rate in the electron beam was quite high the treatment time was not increased excessively.

More recently, a robot mounted linear accelerator has been developed. This device has the advantage of a high degree of freedom since the computer controlled dynamic dose delivery is performed by a robot. However, it requires very long irradiation times for large target volumes since the beam is narrow $(<40 \mathrm{~mm})$ and the dose rate is normal ( $3 \mathrm{~Gy} / \mathrm{min})$. An algorithm for planning and optimizing the application of pencil beams, and also more general types of treatment techniques, has been developed recently $[6,13]$.

\section{Scanning beam therapy}

Radiation therapy is traditionally performed with stationary beams and flattening filters to make the beam uniform. Today, the most rapid and probably safest method for delivering non-uniform beams in real time is based on moving a small elementary electron, photon or proton beam over the patient in much the same way as an electron beam moves in a television set. Beams of this type have been available for almost 10 years at $5-50 \mathrm{MeV}$ racetrack accelerators [21]. Since the elementary, essentially Gaussian electron beams and the bremsstrahlung beams have fairly wide half-widths ( $\geq 12 \mathrm{~mm}$ and $\geq 40 \mathrm{~mm}$, respectively, at the central axis of the beam), a multileaf collimator has to be used for spatial modulation when a higher geometric precision is required. Despite this problem, scanned beams are very useful and in many cases sufficient, at least for beam compensation. When combined with dynamic multileaf collimation, a very fast and flexible dose delivery is possible which is ideal for few-beam, non-uniform, generalized conformal therapy with treatment times of the order of a few minutes in most cases. The scanning system is based on the optical properties of the last bending magnet such that the first scanning magnet deflects the beam in the bending plane of a rotary gantry and a second magnet deflects the beam in and out of the bending plane (Fig. 4, bottom panel). The rotary gantry is also equipped with a detector array for transmission imaging of
Fig. 4. Comparison of six different methods for delivering non-uniform doses. The profiles (intensity versus position) of the beams are indicated together with a schematic of the beam manipulation arrangement and an estimate of the standard treatment time, in minutes, for uniform dose delivery to the target volume. Only the lower three methods allow dynamic beam shaping, but with greatly varying treatment times.

the patient to allow a full comparison between diagnostic and radiotherapeutic computed tomography images so that the patient can be positioned accurately. The energy fluence of the bremsstrahlung beam is obtained by convoluting the scanning density with the elementary bremsstrahlung beam kernel; the therapeutic beam is finely shaped by collimation using, for instance, multileaf collimation.

Dynamically scanned proton beams will probably represent the ultimate in radiation therapy when a high geometrical precision is required. This is because the penumbra of the pencil beam is so narrow that additional collimation is not required [22] and the finite proton range protects tissues beyond the tumour.

\section{References}

[1] I. Lax \& A. Brahme, Radiology 145 (1982) 473; T. Bortfeld et al., Phys. Med. Biol. 35 (1990) 1423; T. Holmes et al., Int. J. Radiat. Oncol. Biol. Phys. 20 (1991) 859 .

[2] A. Brahme in Radiation Therapy Physics, Ed.: A.R. Smith (Springer, Verlag, 1995) 209.

[3] A. Brahme, Accomplishments in Cancer Research 1991, Eds: J.G. Fortner \& J. E. Rhoads (General Motors Cancer Research Foundation, 1992) 265 .

[4] A. Brahme \& A.K. Agren, Acta Oncol. 26 (1987) 377; P. Kallman, B.K. Lind \& A. Brahme, Phys. Med. Biol. 37 (1992) 871.

[5] A. Brahme, B. Lind \& P. Källman, Physica Medica 6 (1990) 53 .

[6] A. Gustafsson, B.K. Lind \& A. Brahme, Med. Phys. 21 (1994) 343.

[7] W.V. Mayneord \& R. Davison, Br. J. Radiol. Suppl. 2 (1950) 150, 197; A. Brahme, Radiother. Oncol. 12 (1988) 129.

[8] A. Brahme, J.-E. Roos \& I. Lax, Phys. Med. Biol. 27 (1982) 1221.

[9] A.M. Cormack, Int. J. Radiat. Oncol. Biol. Phys. 13 (1987) 623; A.M. Cormack \& R.A. Cormack, Int. J. Rad. Qncol. Biol. Phys. 13 (1987) 1921; A.M. Cormack \& E.T. Quinto, Int. J. Imag. Syst. Technol. 1 (1989) 120

[10] A.M. Cormack, Int. J. Imag. Syst. Technol. 6 (1995) 2.

[11] B.K. Lind \& A. Brahme, Phys. Med. Biol. 37 (1992) 891.

[12] T.R. Bortfeld \& A.L. Boyer, Int. J. Imag. Syst. Technol. 6 (1995) 62.

[13] S. Söderstrom, A. Gustafsson \& A. Brahme, Radiother. Oncol. 29 (1993) 148; A. Gustafsson et al., Med. Phys, 22 (1995) 1141.

[14] S. Söderström, A. Gustafsson \& A. Brahme, Int. J. Imag. Syst. Technol. 6 (1995) 91.

[15] P. Kallman et al., Phys. Med. Biol. 33 (1988) 1291.

[16] D. W. Miller in Optimization of Cancer Radiotherapy; Eds.: B.R. Paliwal, D.E. Herbert \& C.G. Orton, AAPM Symp. Proc. No. 5 (Amer. Inst. of
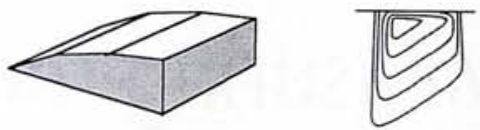

Wedge filters (1.1-2 min)
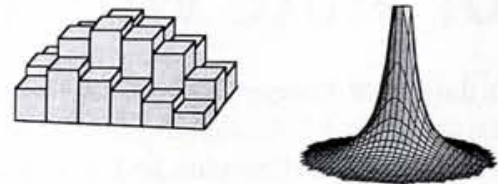

Compensating filters or bolus (1.0-1.5 min)
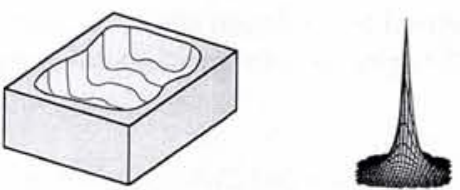

Transmission blocks (1.1-1.5 min)
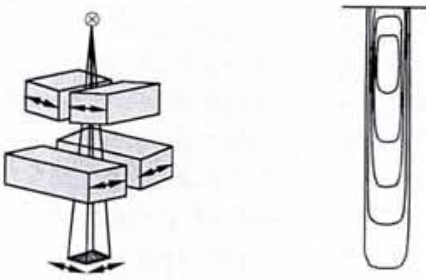

Dual dynamic asymmetric jaw pairs ( $>20 \mathrm{~min}$ )
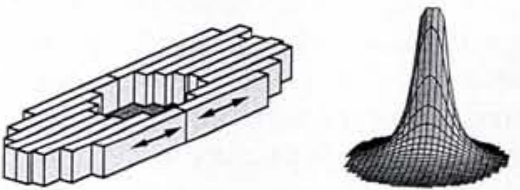

Dynamic multileaf collimation (1.5-2.0 min
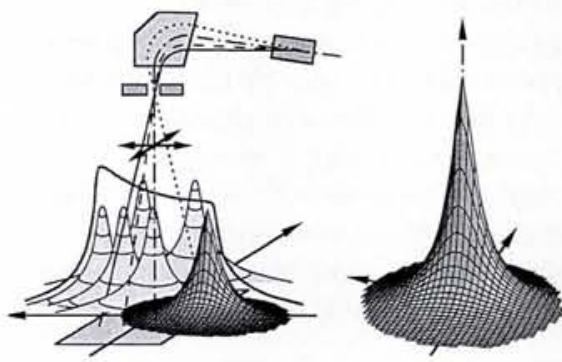

Scanned elementary beams $(0.5-1.0 \mathrm{~min})$

Physics, NY, 1985); N. Ulsø \& A. Brahme, Proc. Joint US-Scandinavian Symp. on Further Directions of Computer-aided Radiation Therapy, San Antonio, TX; Ed.: S. Zink (Nat. Cancer Institute, 1988). [17] A. Brahme, Int. J. Radiat. Oncol. Biol. Phys. 25 (1993) 373.

[18] P. Kallman, A. Agren \& A. Brahme, Int. J.

Radiat. Biol. 62 (1992) 249.

[19] M.P. Carol, Int. J. Imag. Syst. Technol 6 (1995) 56.

[20] T.R. Mackie et al., Int. J. Imag. Syst. Technol. 6 (1995) 43.

[21] A. Brahme, Acta Oncol. 26 (1987) 403; M. Karlson, H. Nyström \& H. Svensson, Med. Phys. 19 (1992) 307; ibid. 20 (1993) 143.

[22] A. Brahme, P. Kallman \& B. Lind, Proc. NIRS Int. Workshop on Heavy Charged Particle Therapy \& Related Subjects, Eds: A. Itano \& T. Kanai (Chiba, Japan, 1991) 124. 